\title{
Automated stimulus intensity control
}

\author{
LARRY WALLNAU and NORMAN GREENFELD \\ State University of New York at Albany, 1400 Washington Avenue, Albany New York 12222
}

This paper describes an apparatus permitting the study of preference for illumination in a barpress task. It is used with a two-bar rat chamber.

Apparatus was constructed which permits the study of preference for illumination with a barpress task. It was designed to be used with a two-bar rat chamber (Grason-Stadler 1111-P), such that the operation of one bar would lead to a gradual, continuous increment and the operation of the other bar would lead to a gradual, continuous decrement in illumination.

The apparatus consists of a clutch-operated, bidirectional motor (Hurst Model PC-DA, $115 \mathrm{~V}, 60 \mathrm{~Hz}$, $2 \mathrm{rpm})$ coupled to the shaft of a variable autotransformer (General Radio Company, Variac Model W5). The output of the autotransformer can operate a light bulb (rated in $\mathrm{V} \mathrm{ac}$ ), the light intensity being proportional to the voltage output of the transformer secondary.

Figure 1 is a diagram of the basic arrangement of the motor and autotransformer in the mounting frame. The dimensions of the mounting frame, although provided in the diagram, are not critical. The motor shaft, however, should be centered as best as possible above the shaft of the autotransformer. Mounting templates for the motor and autotransformer, as well as the necessary dimensions, are provided by the respective manufacturers. When coupled and wired properly, the motor and consequently the autotransformer will rotate in one of two directions, depending on which bar is pressed. The bars in the test chamber and the motor were programmed with Grason-Stadler Series 1200 control equipment; electro-mechanical control equipment may also be used.

Two Grason-Stadler 1216A timers regulate the duration of the motor operation in either direction. For example, if the left bar is pressed, the motor turns counterclockwise for a period of time (e.g., $3 \mathrm{sec}$ ), and produces a decrement in stimulus intensity. When the right bar is pressed, the motor turns clockwise for the same amount of time and produces an increment in light intensity. The timers allow a variety of settings for duration of motor rotation. A selection of useful circuit diagrams are provided with the autotransformer and the Hurst motor. On the autotransformer, Terminal Connections 2 and 4 are for line current and 3 and 4 for load.

Requests for reprints should be sent to Norman Greenfeld, Department of Psychology, State University of New York at Albany, 1400 Washington Avenue, Albany, New York 12222. Complete figures and instructions are available at no charge.
The grounding of Terminal 4 is optional. The output of the autotransformer may also be connected to a stepdown transformer for the control of devices requiring less voltage.

Figure 2 shows the circuit diagram employed to connect the motor to the control apparatus. The GrasonStadler 1203 relay box operates and determines the direction of the motor. The relays are in turn operated via the Grason-Stadler Subject Station Interface (Model 1209). Color coded wires from the motor and from the Subject Station Interface are labeled where necessary. Coupling the motor shaft to the autotransformer can be accomplished by a variety of methods. In this case, a fork-shaped bracket on the motor shaft straddles a T-shaped bracket that is affixed to the autotrans-
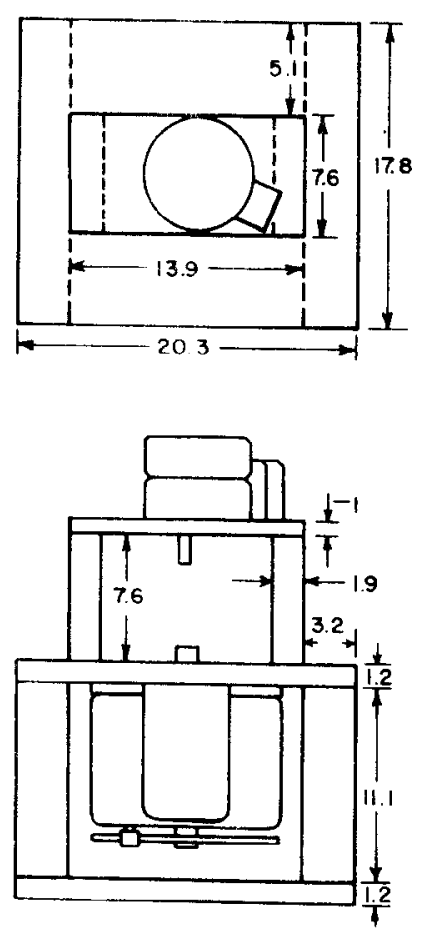

Figure 1. Arrangement of motor and autotransformer in mounting frame. 


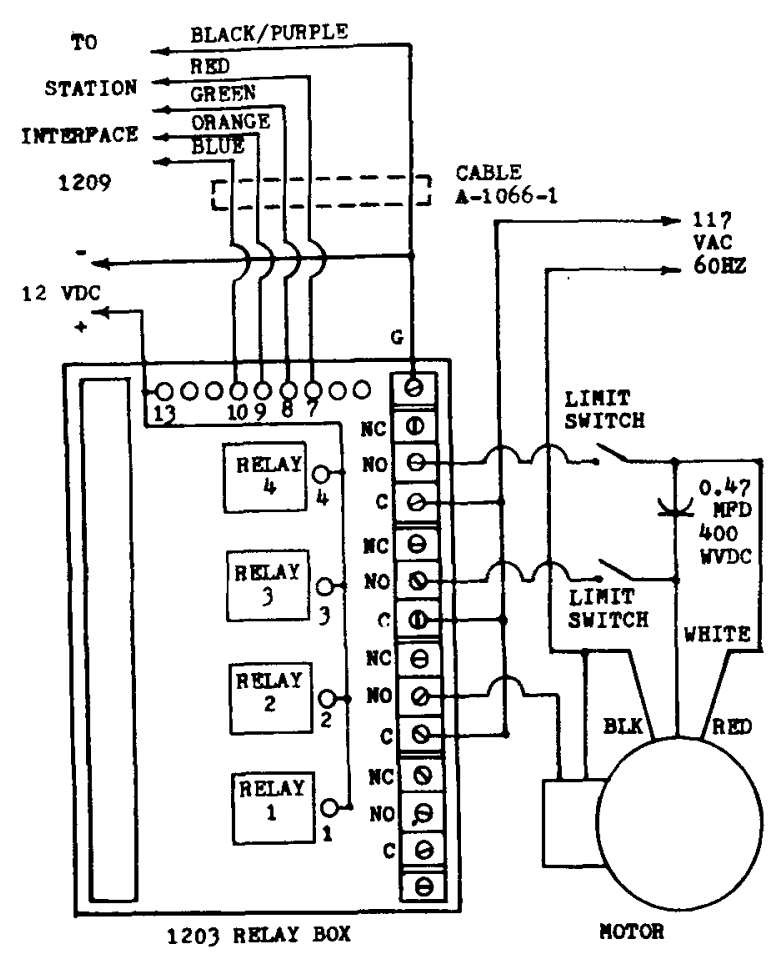

less critical. The coupling shafts consist of metal, plastic, and Plexiglas bonded by a suitable epoxy (EP 12 epoxy, Magic American Chemical Corporation).

To prevent motor and coupling overstrain, limit switches were devised consisting of two microswitches mounted so that as the autotransformer approached one of its limits, a microswitch was depressed and contact broken, preventing the motor from advancing further. Once the motor is activated to turn in the opposite direction, it can once again be made to advance in the original direction.

The brush track on the bottom of the autotransformer has a contact-brush assembly which protrudes from the track. This assembly works as a cam to trip a microswitch positioned on the base of the mounting frame. Exact placement of the microswitches depends on the distance required to trip the switch lever to break contact, and on the points at which one wants to set limits for the variation of the Variac.

The apparatus has been used in pilot studies on preference for illumination in rats. It has been used for long periods, typically $24 \mathrm{~h}$ per rat and 4 to 5 days per week, and its operation has been smooth and reliable.

Figure 2. Circuit diagram.

former shaft, which functions as a universal joint, thus making the alignment of the two different sized shafts
(Received for publication May 28, 1975; revision received August 2, 1975.) 